\title{
Management of Crown Fractures by Reattachment with Minimal Intervention Approach
}

\author{
V Susila Anand ${ }^{1}$, Usha Somasundram ${ }^{2}$
}

\begin{abstract} and translucency of the surface. of maxillary first molar. Keywords: Flowable composites, Fracture, Reattachment.

Journal of Operative Dentistry and Endodontics (2020): 10.5005/jp-journals-10047-0093

\section{INTRODUCTION}

In dental practice, traumatic injuries are the most disturbing emergencies and it is a common challenge for dental professionals because there is ambiguity in choosing the right protocol. It also affects the social and psychological well-being of children.
\end{abstract}

Aim: The case series aims to manage dental trauma by reattaching with the patient's own tooth fragment.

Background: Crowns of anterior teeth and posterior teeth break due to trauma and biting hard objects. Reattachment of fractured tooth fragments by various techniques offers many advantages over other treatments, especially restoration of the esthetics, function, shape, texture,

Case description: The present case series describes three clinical cases of reattachment in which two were done in permanent maxillary central incisors with an uncomplicated and complicated crown fracture and the third case was reattachment of fractured mesiopalatal cusp

Clinical significance: Besides being conservative, fragment reattachment evokes an immediate positive psychological response from the patient.

\section{BACKGROUND}

In dental trauma, coronal fracture of anterior teeth is more common and affects mainly children and young adults. Trauma to the permanent incisors represents $18-22 \%$ of all dental traumatic injuries, among which $96 \%$ involve maxillary central incisors. ${ }^{1}$

The etiological factors of crown and crown root fractures in the permanent teeth are fall (40\%), contact sports (20\%), while automobile accidents, foreign body striking the teeth constitute the rest. ${ }^{2}$

Uncomplicated crown fracture is more common than complicated crown fracture. The extent of dental trauma on the supporting tissues are directly proportional to the impact energy. A blow with a low-speed causes greater damage to the supporting structures, but less commonly dental fractures. On the contrary, impacts at high speed result in a higher incidence of dental fractures, but with less damage to the supporting structures. ${ }^{3}$

To determine the treatment, position of fracture line is an important factor, especially when the supporting gingival tissue is compromised. ${ }^{4,5}$ Management of the fracture by reattaching the fragment with adhesive restorative materials and bonding agents offers advantages, such as, the re-establishment of esthetics, function, shape, texture shade, and original alignment of the teeth. ${ }^{6}$

There are several factors that affect the treatment and prognosis of tooth fractures, like biological width, position of fracture line, pulpal involvement, occlusion, the esthetic need of the patient concerned, whether the fractured fragment is preserved and restorability of the tooth. ${ }^{6}$ Hence, the main aim of all treatment measures must be the preservation of dental tissue, rehabilitate

\begin{abstract}
1,2Department of Conservative Dentistry and Endodontics, Madha Dental College and Hospital, Chennai, Tamil Nadu, India

Corresponding Author: V Susila Anand, Department of Conservative Dentistry and Endodontics, Madha Dental College and Hospital, Chennai, Tamil Nadu, India, Phone: +91 7550022397, e-mail: drsusila2000@gmail.com

How to cite this article: Anand VS, Somasundram U. Management of Crown Fractures by Reattachment with Minimal Intervention Approach. J Oper Dent Endod 2020;5(1):56-61.

Source of support: Nil

Conflict of interest: None
\end{abstract}

the natural esthetics of traumatized teeth, and maintenance of the arch integrity. Literature has witnessed the evolution of different techniques for the reconstruction of injured teeth. Conventionally, composite restorations and post-and-core supported prosthesis are the most commonly used modalities. ${ }^{1}$

Using the tooth fragment when available, for reattachment is the most conservative option for restoration of such tooth. It involves the "minimal intervention" and "biological restoration" concept, which aims to achieve maximum preservation of the natural tooth structure and esthetics. ${ }^{7}$

In 1964, Chosak and Eidelman pioneered this procedure using a cast post and conventional cement for reattaching an anterior crown fragment. The adhesive restorative materials and bonding agents enable the clinician to use the patient's own fragment to restore the fractured tooth more often nowadays. Acid etch technique in reattachment was first used by Tennery. This technique was introduced at the end of the 1970s. ${ }^{8}$

Various techniques are currently being used for the reattachment of the fragments of a fractured tooth. Some of these are circumferential bevel, chamfer, overcontour, lingual bevel, simple reattachment, internal dentin groove, internal bevel of labial enamel, fiber reinforced composite post (FRC), etc.

() The Author(s). 2020 Open Access This article is distributed under the terms of the Creative Commons Attribution 4.0 International License (https://creativecommons. org/licenses/by-nc/4.0/), which permits unrestricted use, distribution, and non-commercial reproduction in any medium, provided you give appropriate credit to the original author(s) and the source, provide a link to the Creative Commons license, and indicate if changes were made. The Creative Commons Public Domain Dedication waiver (http://creativecommons.org/publicdomain/zero/1.0/) applies to the data made available in this article, unless otherwise stated. 
This case series describes the management of three cases in which two were complicated crown fracture and one uncomplicated fracture by reattachment of the fractured tooth fragment.

\section{Case Description}

\section{Case 1}

A 14-year-old male patient reported to the Department of Conservative Dentistry and Endodontics with fractured maxillary anterior tooth. The tooth had fractured 12 hours ago on account of a fall. Patient complained of mild sensitivity in 21. Clinical and radiographic examination revealed Ellis class II fracture in 21 and Ellis class I fracture involving mesial incisal enamel edge in 11 (Figs $1 \mathrm{~A}$ and B). The oblique fracture line in 21 was supragingival both labially and palatally. No other fracture in the root and healthy periapical tissues was detected. The patient had brought the fragment in handkerchief (Fig. 1C). The treatment plan decided for the patient was reattachment of the fragment. Fragment was cleaned with water and stored in saline for 30 minutes for rehydration. The fragment was checked for approximation and rubber dam isolation done. Two vertical internal dentinal grooves of $2.0 \mathrm{~mm}$ depth and 1.5 $\mathrm{mm}$ width were bilaterally made within the dentin of the fragment, $1 \mathrm{~mm}$ away from the dentinoenamel junction using high-speed \#4 round carbide bur (Fig. 1D).

The fractured fragment and the remaining tooth structure were etched with $37 \%$ orthophosphoric acid gel for 20 seconds (Figs $1 \mathrm{E}$ and F). After thorough rinsing and drying, adhesive (Te-Econom bonding agent IVOCLAR) was placed on both tooth fragment and tooth structure and air thinned and light cured for 10 seconds. Tooth fragment was reattached using Te-Econom low-viscosity flowable resin cement (A2shade) and light cured. Excess composite material was removed, finished, and polished (Fig. 1G). The patient was advised dietary, oral hygiene instructions.

\section{Case 2}

A 21-year-old male patient reported to the Department of Conservative Dentistry and Endodontics with fractured maxillary right central incisor. Clinical and radiographic examination revealed Ellis class III fracture with involvement of the pulp in 11 and Ellis class II fracture in 21 . The fracture line of 11 was located supragingivally on the labial side, whereas it extended subgingivally on the palatal side (Fig. 2A). No other hard or soft tissue injury but for the tooth fracture was observed. The patient carried the broken tooth fragment that was stored in water. The trauma had occurred 24 hours prior to the patient's visit and the fragment did not show any change in color (Fig. 2B).

The fragment was checked for fit (Fig. 2C). After completing the root canal treatment (Fig. 2D), crown lengthening was done to expose the fracture site in the palatal aspect (Fig. 2E). Then, post space was prepared using Peeso reamers (up to size 4) and FRC post of $1.2 \mathrm{~mm}$ diameter was luted with resin cement extending the post $2.5 \mathrm{~mm}$ extracoronally for the fragment to engage (Fig. $2 \mathrm{~F})$. A vertical channel was created in the fragment internally in the dentin of about $1.5 \mathrm{~mm}$ width and $2.5 \mathrm{~mm}$ depth to adapt to the post (Fig. 2G). The fragment and the tooth with FRC post was coated with Tetric $\mathrm{N}$ bond universal self-etch adhesive and light cured for 40 seconds. Then, the fragment was reattached to the tooth using Tetric N Flow flowable composite, excess composite removed and light cured for 40 seconds on buccal and palatal aspect (Figs $2 \mathrm{H}$ to J). Postoperative radiograph and photograph showed the fit and adaptation of the fragment (Figs $2 \mathrm{~K}$ and $\mathrm{L}$ ). Patient was instructed with appropriate postoperative care.

\section{Case 3}

A 27-year-old male patient reported to the Department of Conservative Dentistry and Endodontics with pain in his upper left posterior region. Clinical and radiographic examination revealed the fracture of mesiopalatal cusp of maxillary left first molar (tooth no-26) involving the pulp in which the fracture extends subgingivally and an intact fragment held in place by gingival tissue (Figs $3 A$ and $B$ ).

Under rubber dam isolation, access opening and pulp extirpation were done and closed dressing given (Fig. 3C). The fragment was prepared (cleaned with pumice slurry) to receive the application of adhesive and composite resin. Tetric $\mathrm{N}$ bond universal self-etch adhesive was applied using applicator tip (Fig. 3D) and then light cured for 40 seconds, then a Tetric N Flow flowable composite was placed between the tooth and the fragment and finger pressure applied to reattach the fragment and light cured for 40 seconds (Figs 3D and F).

In the second visit, endodontic treatment was completed (Fig. 3G). In the third visit, crown lengthening was done in the palatal aspect to expose the fractured site, then composite entrance filling given and crown preparation made for metal ceramic crown in 26 (Fig. 3H). Crown cementation was done using type I GIC in the fourth visit (Fig. 3I). The patient was instructed with appropriate postoperative and oral hygiene care.

\section{Discussion}

Composite resin restoration is the esthetic treatment of choice for fractured anterior teeth. Though these restorative materials do not structurally resemble enamel or dentin, their advanced formulations possess optical properties, such as, translucency, opalescence, fluorescence, and surface gloss. However, synthetic materials cannot match nature in quality or stability. ${ }^{9}$

Also, wear of enamel of the opposing dentition by composite resin is higher. But, when the fragment of the fractured tooth used, the rate of abrasiveness and wear is the same as that for the intact tooth. In addition, it is less time-consuming, reducing the overall treatment cost. ${ }^{10}$

Due to the protection of the natural tooth structure, it also gives a psychologically positive response. In the case of children and adolescents, both patient and parents are satisfied because original fragment is used in the restoration. ${ }^{11}$

Reis et al. studied the fracture resistance of teeth when reattached. They found fracture resistance of simple reattachment was very low of about $37.1 \%$, whereas buccal chamfer, superficial overcontouring, and internal groove placement showed the recovery of $60.6,97.2$, and $90.5 \%$ of fracture resistance, respectively. "Over contour" and "internal dentinal groove" have been found to yield better results in other studies also. Hence, in the first case, internal groove placement was done to improve the fracture resistance of traumatized tooth.

In the second case, fiber-reinforced post was used for reattaching the fractured fragment with the tooth after completion of endodontic treatment was planned as FRC posts have several advantages over metal posts. Their principal advantages are that they are tooth colored, passive, bend along with dentin upon being flexed as their elastic modulus is similar to that of dentin. ${ }^{13}$ Reattachment using the FRC post which is bonded into the root canal enhances the retention of the crown's fractured fragment. 

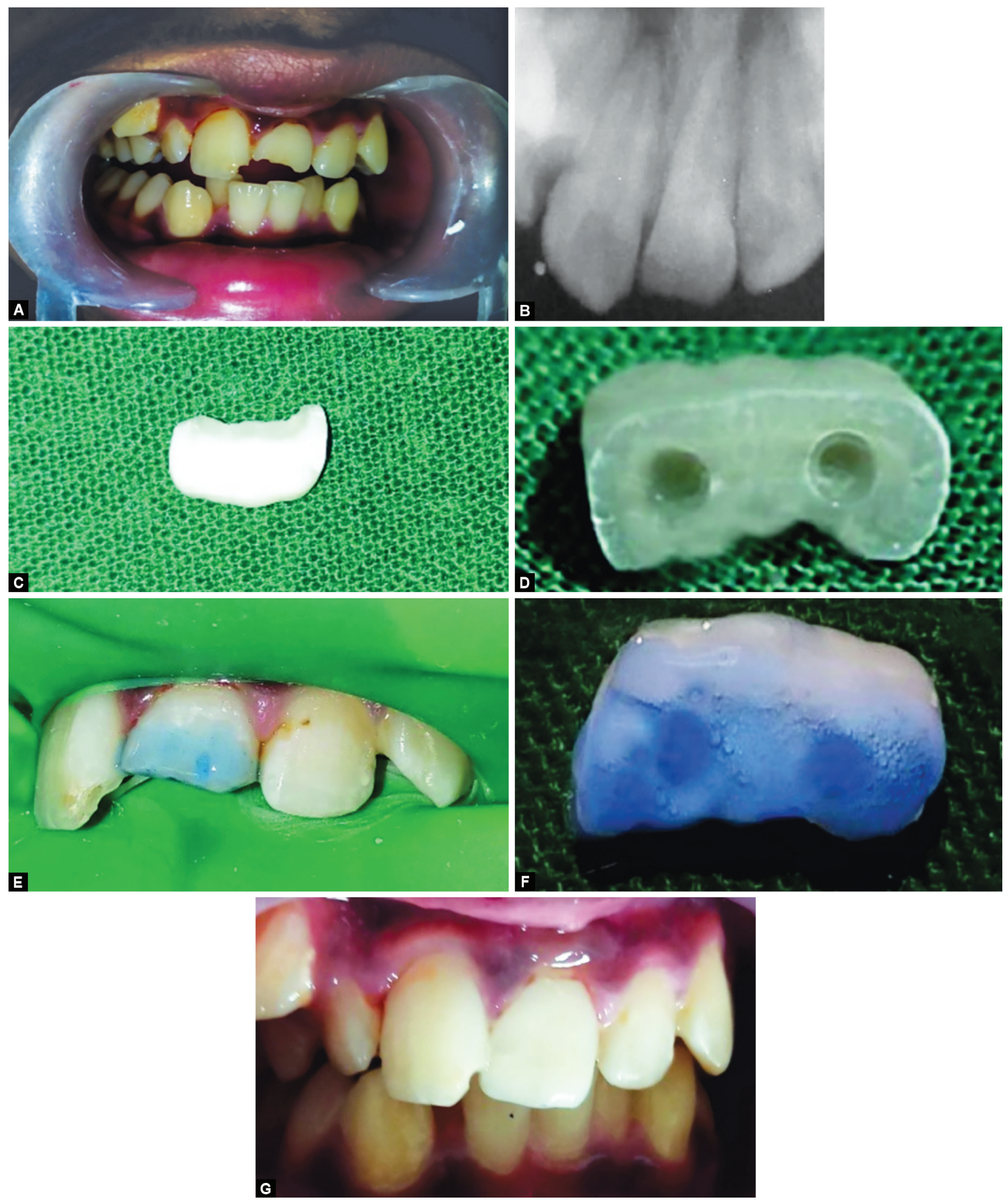

Figs 1A to G: (A) Preoperative clinical photograph; (B) Preoperative intraoral periapical radiograph; (C) Broken tooth fragment; (D) Two vertical internal dentinal grooves; (E) Acid etching done on intact tooth; (F) Acid etching done on fragment; (G) Postoperative clinical photograph

The increase in fracture resistance is due to the combination of elastic and adhesive characteristics. Thus, both the tooth and post move and flex as a single unit, which ensures the favorable stress distribution. ${ }^{14}$
In the second case presented here, the extension of the fracture line was subgingival palatally. However, as the fracture was supraalveolar, sufficient access, visibility, and isolation were achieved by gingivectomy. ${ }^{15}$ Hence, the tooth fragment reattachment allows 

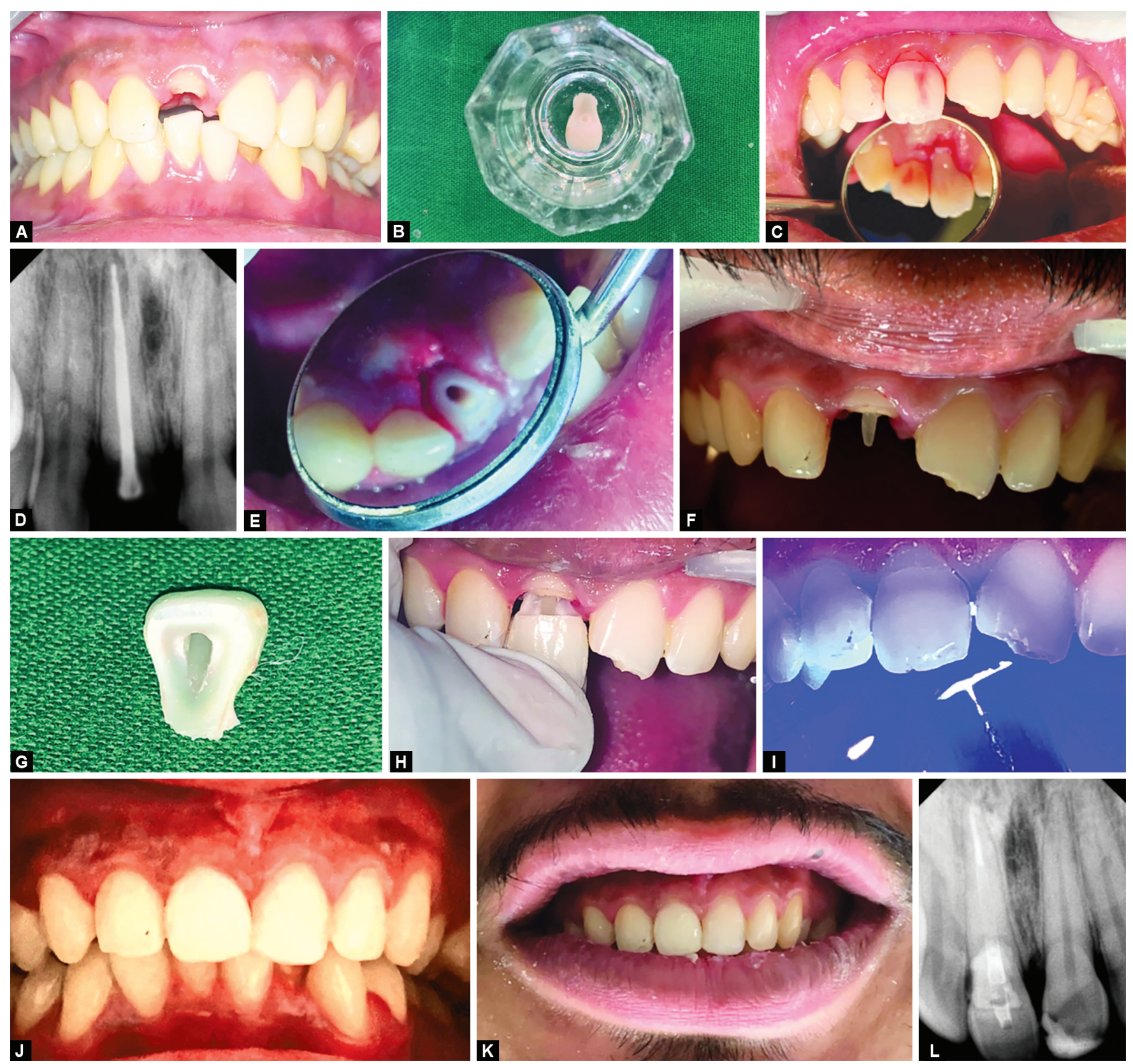

Figs 2A to L: (A) Preoperative clinical photograph; (B) Broken tooth fragment; (C) Fragment checked for fit; (D) Intraoral periapical radiograph of root canal treatment; (E) Crown lengthening done; (F) FRC post luted; (G) Vertical hole placed; $(\mathrm{H})$ Reattachment of fragment; (I) Excess removed and light cured; $(\mathrm{J})$ Finishing and polishing done; (K) Postoperative clinical photograph; (L) Postoperative intraoral periapical radiograph

restoration of the tooth and conservation of the remaining tooth structure to the maximum possible extent. The use of patient's own tooth fragment clearly eliminated the problems of unmatched shades, abrasion, poor contour, difficulty in obtaining polished surface reproduction. ${ }^{16}$ Furthermore, the soft tissue response to any other restoration would be far more adverse than the natural tooth fragment and hence this reattachment should be the primary option for treating subgingival fractures.

In self-etching bonding agents, dentin is prepared to react adhesively by modifying the smear layer with the help of acidic primers. Jayasheel et al. found that the shear bond strength of Tetric $\mathrm{N}$ bond universal adhesive was higher than Single Bond Universal and ClearFil SE. ${ }^{17}$
Rangappa et al. compared the shear bond strength of flowable composite and concluded that Tetric N Flow flowable composite was found to have higher bond strength compared to Constic, Dyad ${ }^{\mathrm{TM}}$-flow. ${ }^{18}$ Tetric N-Flow is based on nano-optimized technology. Hence in the second and the third case, Tetric $\mathrm{N}$ bond universal adhesive and Tetric N Flow flowable composite were used for reattaching the fragments to the fractured teeth.

In the third case, this reattachment technique was used as it is operator-friendly and conservative. Furthermore, as mentioned earlier, it is relatively faster and hence cost-effective. Also, it improves resistance to staining and abrasion than the composite build-up. In this case report, to reinforce the cusps of pulpless teeth in which the functional mesiopalatal cusp is weakened by fracture 

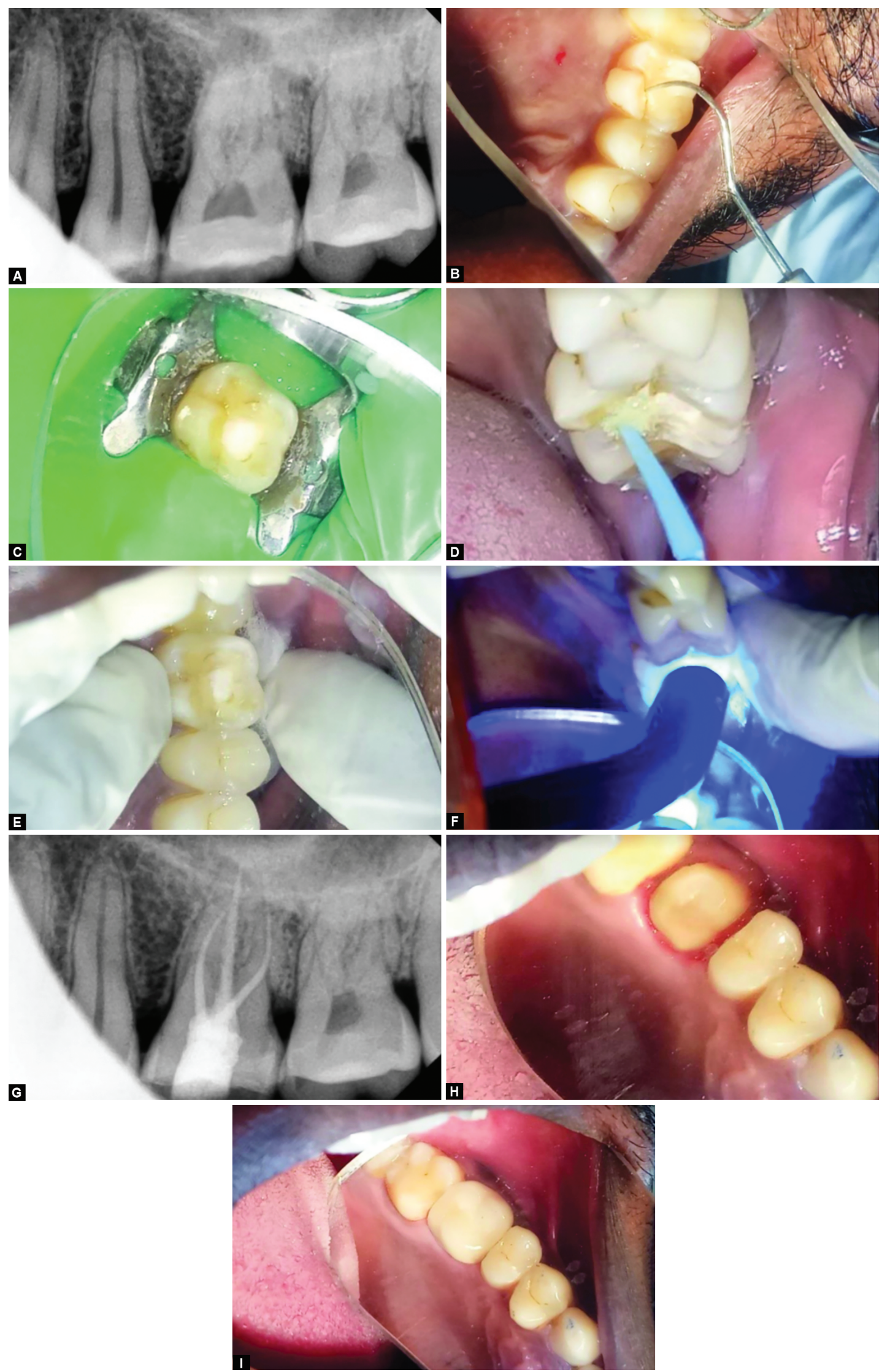

Figs 3 A to I: (A) Preoperative intraoral periapical radiograph; (B) Preoperative clinical photograph; (C) Access opening done; (D) Self-etch adhesive applied; (E) Fragment reattached; (F) Light cured; (G) Intraoral periapical radiograph of root canal treatment; (H) Crown lengthening and crown preparation; (I) Crown cementation postoperative photograph 
and enhance longevity postendodontic treatment, full crown rehabilitation was done to withstand forces of everyday mastication in a strategic tooth with a fractured functional cusp.

\section{Conclusion}

This case series shows the advantages of minimally invasive treatment to improve the prognosis of traumatized teeth. Emphasis is being given to those techniques that restore biological width. With the materials available today, when paired with a suitable technique, predictable esthetics can be achieved. While treating children and adolescents, esthetics is their primary concern. Thus, the fragment reattachment is an excellent procedure to obtain maximal function and esthetics without sacrificing more tooth structure.

\section{Clinical Significance}

The technique described here offers substantial benefits for both the operator and the patient alike. When the fractured fragment of the tooth is used, the result is esthetically more appealing. When there is a fracture which is below the alveolar bone height then other techniques like orthodontic extrusion or surgical crown lengthening should be considered.

\section{References}

1. Abdulkhayum A, Munjal S, Babaji $P$, et al. In vitro evaluation of fracture strength recovery of reattached anterior fractured tooth fragment using different re-attachment techniques. J Clin Diagn Res 2014;8(3):208-211. DOI: 10.7860/JCDR/ 2014/7161.4164.

2. Londhe SM, Garge HG, Sudeep S. Reattachment of crown fragment for immediate esthetic. Clin Pract 2008;64(4):387-390. DOI: 10.1016/ S0377-1237(08)80039-1.

3. Lauridsen E, Hermann NV, Gerds TA, et al. Pattern of traumatic dental injuries in the permanent dentition among children, adolescents and adults. Dent Traumatol 2012;28(5):358-363. DOI: 10.1111/j.16009657.2012.01133.x.

4. Martos J, Marques MM, Katrein CR, et al. Transurgical re-attachment of coronal fragment in anterior-fractured tooth. Eur J Gen Dent 2013;2(1):76-79. DOI: 10.4103/2278-9626.106824.

5. Cruz MK, Martos J, Silveira LF, et al. Odontoplasty associated with clinical crown lengthening in management of extensive crown destruction. J Conserv Dent 2012;15:56-60. DOI: 10.4103/0972 0707.92608.

6. Macedo GV, Diaz PI, De O Fernandes CA, et al. Reattachment of anterior teeth fragments: a conservative approach. J Esthet Restor Dent 2008;20(1):5-18. DOI: 10.1111/j.1708-8240.2008.00142.x.

7. Raut AW, Mantri V, Shambharkar VI, et al. Management of complicated crown fracture by reattachment using fiber post: minimal intervention approach. J Nat Sc Biol Med 2018;9(1):93-96. DOI: 10.4103/jnsbm.JNSBM_98_17.

8. Choudhary A, Garg R, Bhalla A, et al. Tooth fragment reattachment: an esthetic, biological restoration. J Nat Sci Biol Med 2015;6(1):205-207. DOI: 10.4103/0976-9668.149123.

9. Goenka P, Sarawgi A, Dutta S. A conservative approach toward restoration of fractured anterior tooth. Contemp Clin Dent 2012;3(Suppl 1):S67-S70. DOI: 10.4103/0976-237X.95109.

10. Goenka P, Marwah N, Dutta S. Biological approach for management of anterior tooth trauma: triple case report. J Ind Soc Pedodon Prev Dent 2010;28(3):223-229. DOI: 10.4103/0970-4388.73791.

11. Yilmaz Y, Zehir C, Eyuboglu O, et al. Evaluation of success in the reattachment of coronal fractures. Dent Traumatol 2008;24(2): 151-158. DOI: 10.1111/j.1600-9657.2007.00532.x.

12. Reis A, Francci C, Loguercio AD, et al. Re-attachment of anterior fractured teeth: Fracture strength using different techniques. Oper Dent 2001;26(3):287-294.

13. Gbadebo OS, Ajayi DM, Oyekunle OO, et al. Randomized clinical study comparing metallic and glass fiber post in restoration of endodontically treated teeth. Indian J Dent Res 2014;25(1):58-63. DOI: 10.4103/0970-9290.131126.

14. Bosso K, Gonini JA, Guiraldo RD, et al. Stress generated by customized glass fiber posts and other types by photoelastic analysis. Braz Dent J 2015;26(3):222-227. DOI: 10.1590/0103-6440201300256.

15. Adanir N, Ok E, Erdek Y. Re-attachment of subgingivally oblique fractured central incisor using a fiber post. Eur J Dent 2008;2(2): 138-141. DOI: 10.1055/s-0039-1697369.

16. Hegde S, Tawani G, Warhadpande M. Use of quartz fiber post for reattachment of complex crown root fractures: a 4 year follow-up. J Conserv Dent 2014;17(4):389-392. DOI: 10.4103/0972-0707. 136519.

17. Jayasheel A, Niranjan N, Pamidi $\mathrm{H}$, et al. Comparative EVALUATION of shear bond strength of universal dental adhesives: an in vitro study. J Clin Exp Dent 2017;9(7):e892-e896. DOI: 10.4317/ jced.53816.

18. Rangappa A, Srinivasulu J, Rangaswamy V, et al. Comparative evaluation of bond strength of self-adhering flowable composites to the dentin prepared with different burs: an in vitro study. J Conserv Dent 2018;21(6):618-621. DOI: 10.4103/JCD.JCD_71_18. 